\title{
Introduction: History of the management of advanced renal cell cancer
}

\author{
Janice P. Dutcher
}

Published online: 14 January 2009

(C) Humana Press Inc. 2009

Renal cell cancer has been one of the more troubling malignancies for its lack of responsiveness to conventional chemotherapeutic agents, over 30 years of drug development. It has also been one of the more fascinating malignancies for its unusual natural history and the frequent presence of multiple paraneoplastic syndromes, occurring either early or late in the course of the illness, and for the rare occurrence of spontaneous regression of metastatic disease.

During the late 1970s and 1980s, evolving interest in stimulating the host immune response as a form of anticancer therapy led to observations of beneficial responses to interferons in patients with advanced renal cell cancer. Subsequently, the teasing out of approaches to stimulate immune cells, T-cells and natural killer cells, led to the identification of interleukin-2 (IL-2) (initially called T-cell growth factor) in this role, and to pre-clinical experiments demonstrating immune activation with this agent. Additional studies in murine systems demonstrated the ability of IL-2 stimulated killer cells to produce tumor regression through treatment with IL2 and activated killer lymphocytes. With the development of recombinant DNA technology, milligram quantities of interferons and interleukins led to the ability to move these agents into largescale clinical trials in patients with malignancies. Interest in immunotherapy for renal cell cancer was initially sparked by its unusual natural history, with sometimes a decade between the time of initial diagnosis and the development of metastatic disease. What is the mechanism

J. P. Dutcher $(\bowtie)$

New York Medical College, 600 East 233rd Street,

Bronx, NY 10466, USA

e-mail: jpd4401@aol.com of this phenomenon? Perhaps innate immunity, but it is still not clear. Additionally, there are anecdotal reports of spontaneous regression of concurrent metastatic disease following nephrectomy. Again, what the mechanism is remains unclear. Nevertheless, these observations combined with technological advancements led to clinical trials of interleukin-2 and interferon in patients with advanced renal cell cancer during the late 1980s and 1990s.

For the first time in the treatment of adenocarcinoma of any organ in adults, rare complete responses following high dose IL2 therapy led to durable remissions in patients with metastatic renal cell cancer. Some of these responses have now lasted more than 20 years. Unfortunately, this is not the most common outcome of treatment. These differences among patients' responses have led to considerable research efforts in studying interactions of immune cells and tumors, including anti-tumor effects, mechanisms of inactivation of immune cells by tumors, and the various components of activation and inactivation of immune response. Additionally, much clinical research evaluated combination cytokine therapy, and attempts to further the effect of IL-2.

In this supplement, we review the current status of treatment of advanced renal cell cancer with high dose IL2, and some of what we have learned about its efficacy, the immune system in cancer treatment, and interactions of these pathways with tumor characteristics, which may lead to better identification of candidates for IL2 therapy. We also suggest future directions for this therapy that has led to some lives being saved through treatment of advanced disease.

The second part of this supplement proceeds into the 21 st century, where "targeted therapy" has become the hope of oncology treatment. Clearly, we are identifying new mechanisms of anti-tumor activity, affecting both 
tumor behavior and interactions with the host, such as inhibiting the new blood vessel formation that allows the tumor to grow and receive nutrients. Over the past 10 years, anti-angiogenesis as an anti-tumor mechanism has come into the mainstream of anti-cancer therapy, in many solid tumors, in conjunction with chemotherapy. The vascular nature of renal cell cancer has made it a tumor of great interest for studying this anti-tumor mechanism as a primary therapeutic approach. Recent clinical trials of multi-kinase inhibitors (sunitinib and sorafenib) and more specific anti-vascular endothelial growth factor receptor (VEGFR) kinase inhibitors (axitinib and pazopanib) as well as the antibody binding specifically to VEGF, bevacizumab, have shown that inhibiting this pathway is a mechanism that is active against advanced renal cell cancer. Again this is not the entire story, because the outcome leads to tumor inhibition and stability, but not to complete regression, and stable disease is not eternally durable.

Therefore, research evaluating other pathways, affecting targets upstream or downstream of or in parallel with VEGFR, is ongoing and necessary. The mammalian target of rapamycin (mTOR) is such an attractive target. While its inhibition has anti-angiogenesis effects, mTOR is central to numerous additional pathways, and its inhibition has effects on cellular metabolism and growth and progression of the cell cycle. Therefore, as we focus on these pathways, the effect of these new agents in treatment of renal cell cancer has been both enlightening and gratifying. For the first time in 20 years, we have new agents that demonstrate significant anti-tumor activity for advanced renal cell cancer. The promise of the future is that further elucidation of these pathways will lead to additional agents that will be effective therapy. Additionally, in the pattern of traditional oncology regimen development, ongoing studies will evaluate the ability to combine these newer agents to enhance anti-tumor activity.
Suddenly, those of us who have been treating renal cell cancer for many years, with some improvements, and many disappointments, find numerous possibilities for these patients, and numerous opportunities for additional research and treatment development. It is indeed an exciting time for the management of this disease as we attempt to identify a treatment algorithm that will allow us to move the field forward. Questions still to be answered include the following: Defining when to start targeted treatment in stable patients (when the expected result is stable disease); who will respond to IL-2 and perhaps have a durable complete response? Are there biologic clues identifying increased disease activity, warranting initiation of treatment? Are there tumor or patient characteristics to define which treatment is most likely to be efficacious? What are the criteria to change therapy? What is the benefit of treating beyond progression when the disease progression velocity has diminished? Should we revisit drugs we have previously used? Is there true resistance to the kinase inhibitors, or can we recycle them? Are combinations of drugs better than sequential therapy, or is the converse true? What other combinations are feasible? Is there synergy between anti-VEGF agents and interferon as suggested by recent trials?

Clearly, there are far more questions than answers at this point in time. The factors that make renal cell cancer a unique disease, and its management unique among other adult malignancies, also suggest that renal cell cancer treatment investigators have much additional work to do. The interaction of renal cell cancer with these quite disparate types of therapies also suggests that there are additional unique features yet to be identified. This will continue to make the treatment and management of renal cell cancer an unusual and fascinating clinical problem for years to come. 